\title{
A New Approach for the Control Optimization of an Assembly/Disassembly Mechatronics Line Served by an Autonomous Robotic System
}

\author{
Eugenia MINCA \\ Department of Automation, Computer Science and Electrical Engineering, \\ "Valahia” University of Targoviste, Romania, \\ 18-24 Unirii Boulevard, 130082 Targoviste, ROMANIA, \\ eugenia.minca@gmail.com
}

\begin{abstract}
In this paper a generalized Synchronized Hybrid Petri Nets (SHPN) model for the control of repetitive tasks is presented. It is assumed that the process has both discrete and continuous components but integrated in repetitive tasks series. Generalized SHPN model describe this hybrid system with $\mathrm{N}$ degree of repetitive tasks. The proposal is customized to an assembly/disassembly process of a mechatronics line (A/DML), served by a wheeled mobile robot (WMR) equipped with robotic manipulator (RM). For the A/DML model, the assembly is a conventional process with a specific typology of discrete system events (DES) while the disassembly process (served by WMR), is assimilated with a hybrid process. The hybrid aspect, identified only on the disassembly levels is determined by the mobile robot states with discrete and continuous variation characteristics. The hybrid control system takes into account the distribution of the necessary tasks to perform the disassembly of components by using the robot synchronization with the A/DML. Taking all these aspects into consideration, an optimization approach of time cycle for repetitive processes is proposed.
\end{abstract}

Keywords: assembly/disassembly; manufacturing line; wheeled mobile robot; robotic manipulator; Petri Nets

\section{Introduction}

This paper proposes a generalized Synchronized Hybrid Petri Nets (SHPN) dedicated for the control of a hybrid repetitive process. The tool SHPN is dedicated to control modeling of hybrid systems composed of repetitive tasks series. These repetitive components we define as the elementary operations. The model of entire process requires using specific tools dedicated to discrete or continuous DES model, but adapted for repetitive and synchronized tasks description.

The proposal will be tested for a reversible assembly/disassembly manufacturing line (A/DML) served by wheeled mobile robot (WMR) equipped with robotic manipulator (RM).

In this paper, the concepts of assembly/disassembly tasks are illustrated in SHPN model complies with discrete approach for the elementary assembly/disassembly operations and the continuous approach for displacement of WMR. The considered system is a hybrid one and requires specialized tools for modeling, as in (David and Alla, 2010).The hybrid model is elaborated using the dedicated modeling tool, HPN, described in (Filipescu, et al., 2012) and (Radaschin, et al., 2011). Combining the SED model of the analyzed system with the cyclic and continuous time of the WMR with RM results a SHPN model.
This paper is organized as follows: the description of A/DML served by WMR with RM and SHPN model, in generalized and customized forms, are presented in Section 2; the generalized and customized SHPN formalism is presented in Section 3. Section 4 is reserved to the optimization of the time cycle corresponding to the control of repetitive processes; some conclusion remarks can be found in Section 5.

\section{General Structure of the A/DML Served by WMR with RM.}

The system of reversible assembly/disassembly line served by robotic manipulators mounted on mobile platforms has a dynamics determined both, by events (events supplied by the control sequences of the automation system) and by the interaction with the WMR, which represent the continuous time component of the system.

The assembly/disassembly line is served by a WMR equipped with RM during of disassembly phase. The objective is to make the assembly line reversible, i.e. to allow disassembly. Moreover, the mobile robot is used to carry the disassembled component to proper storage warehouse.

The assembly/disassembly operation can by decomposed into a sequence of elementary assembly tasks coupled in parallel with positioning tasks of work-piece along conveyor, 
as in (Baldwin, et al., 1991; Choi, et al., 1998; Ganget, et al., 2005) and (Iacob and Popescu, 2013). The hybrid disassembly strategy is based on the hierarchical model proposed in (Selinger, et al., 1999; Radaschin, 2011; Radaschin et al., 2012; Kopacek, and Kopacek, 1999) which uses the general representation from Figure 2.
- assembling/storage in warehouses (TPN typology);

- disassembling of damaged product (SPN and TPN typologies);

- $\quad$ service assistance, during disassembling process, performed by the mobile robot equipped with manipulator (THPN typology)

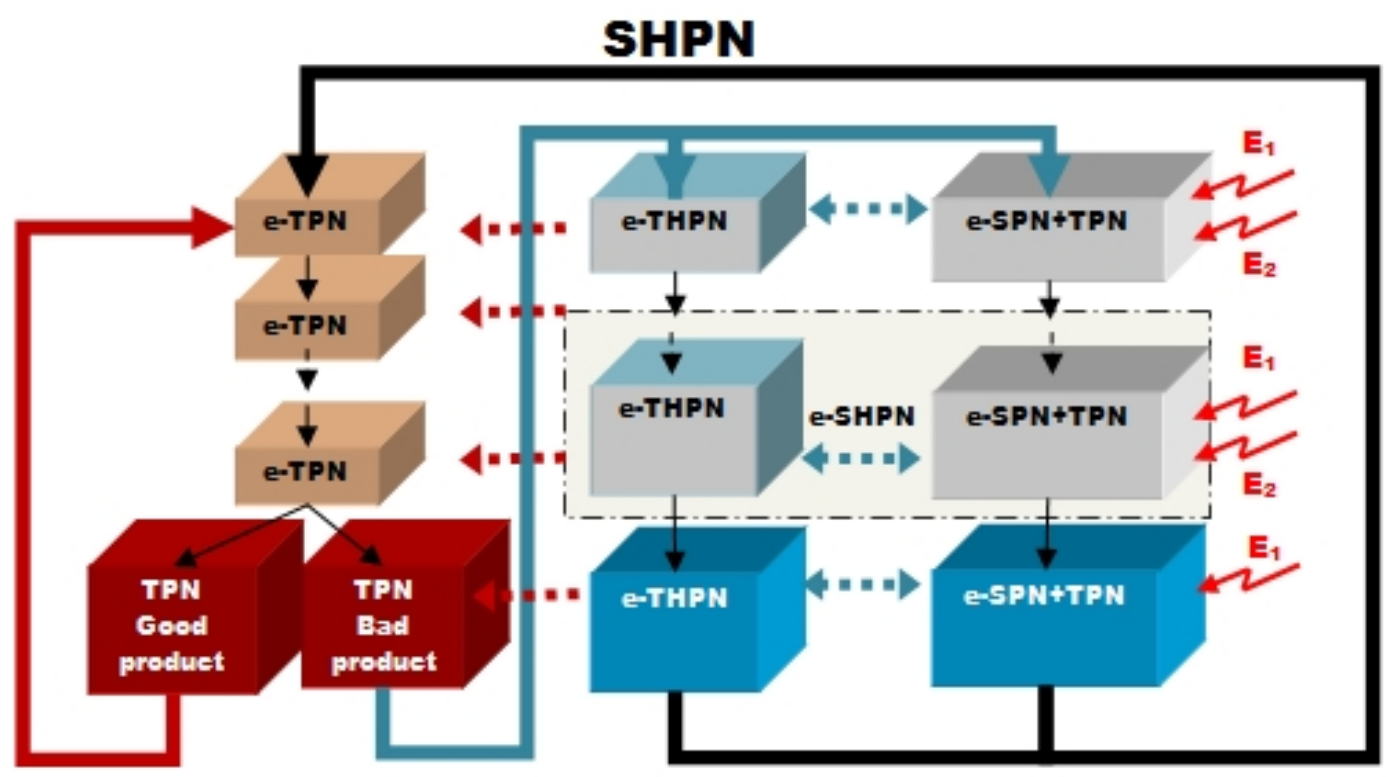

Figure 1. The SHPN representation by blocks with elementary modules:e-TPN for assembly, e-THPN for WMR with RM, e-SPN+TPN for disassembly and e-SHPN for disassembly served by WMR with RM

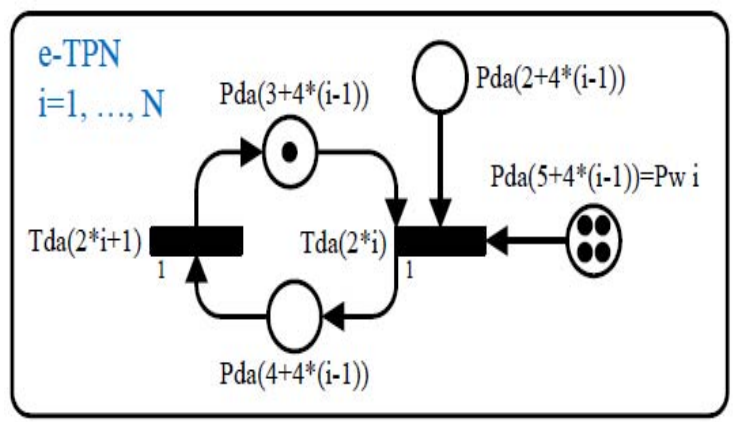

Figure 2. e-TPN model for an elementary assembly operation.

SHPN structure from Figure 1 is obtained by modeling of assembly/disassembly and continuous service assistance, for disassembly operations, performed by mobile platform equipped with manipulator.

The entire model is SHPN type because it is interfaced with external events for synchronization in an approach of modeling/simulation, useful in real-time control. SHPN morphology results by integration three PN models. These models describe the following automatic operations:
$E_{d d(j)}^{1}$ and $E_{d d(j+2)}^{2}$ are external events from the sensors used for line synchronisation with the WMR equipped with RM. $E_{d d(j)}^{1}$ is an external synchronization signal, corresponding to STOPPING line and STARTING disassembly. $\quad E_{d d(j+2)}^{2}$ is an external synchronization signals, corresponding to PICKING UP of disassembled component and STARTING line.

In Figure 2 is represented an elementary TPN model corresponding to an assembly operation. During disassembly process one can identify a repetitive sequence associated to a single disassembly operation and service assistance of WMR equipped with RM. All of these can be modelled with a SHPN, called elementary SHPN, as is represented in Figure 3.

Since the last disassembly operation is no longer necessary line starting to a next disassembly, the SHPN model is different from others and is shown in Figure 4. 


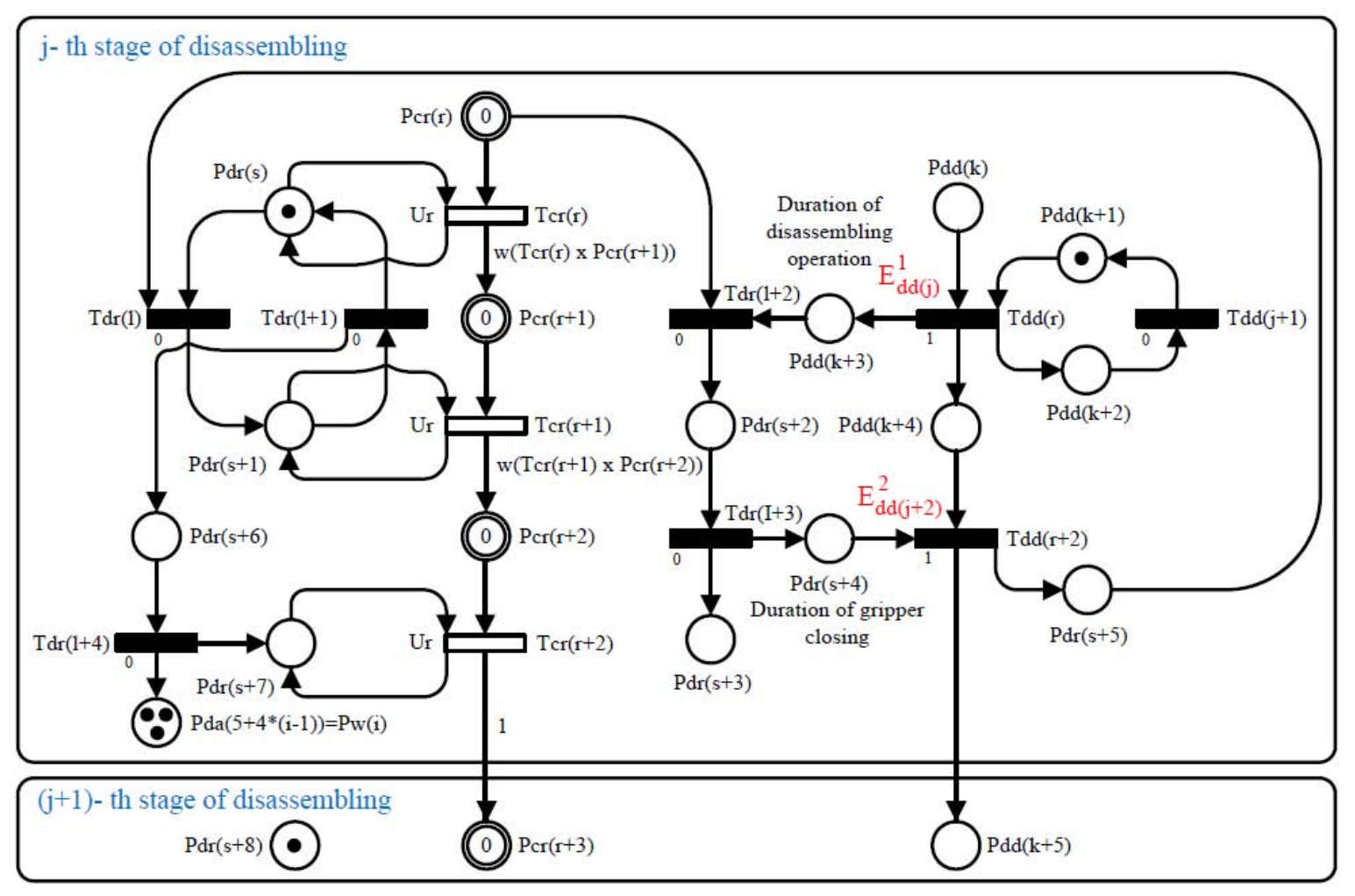

Figure 3. e-SHPN model of j-th elementary disassembly operation.

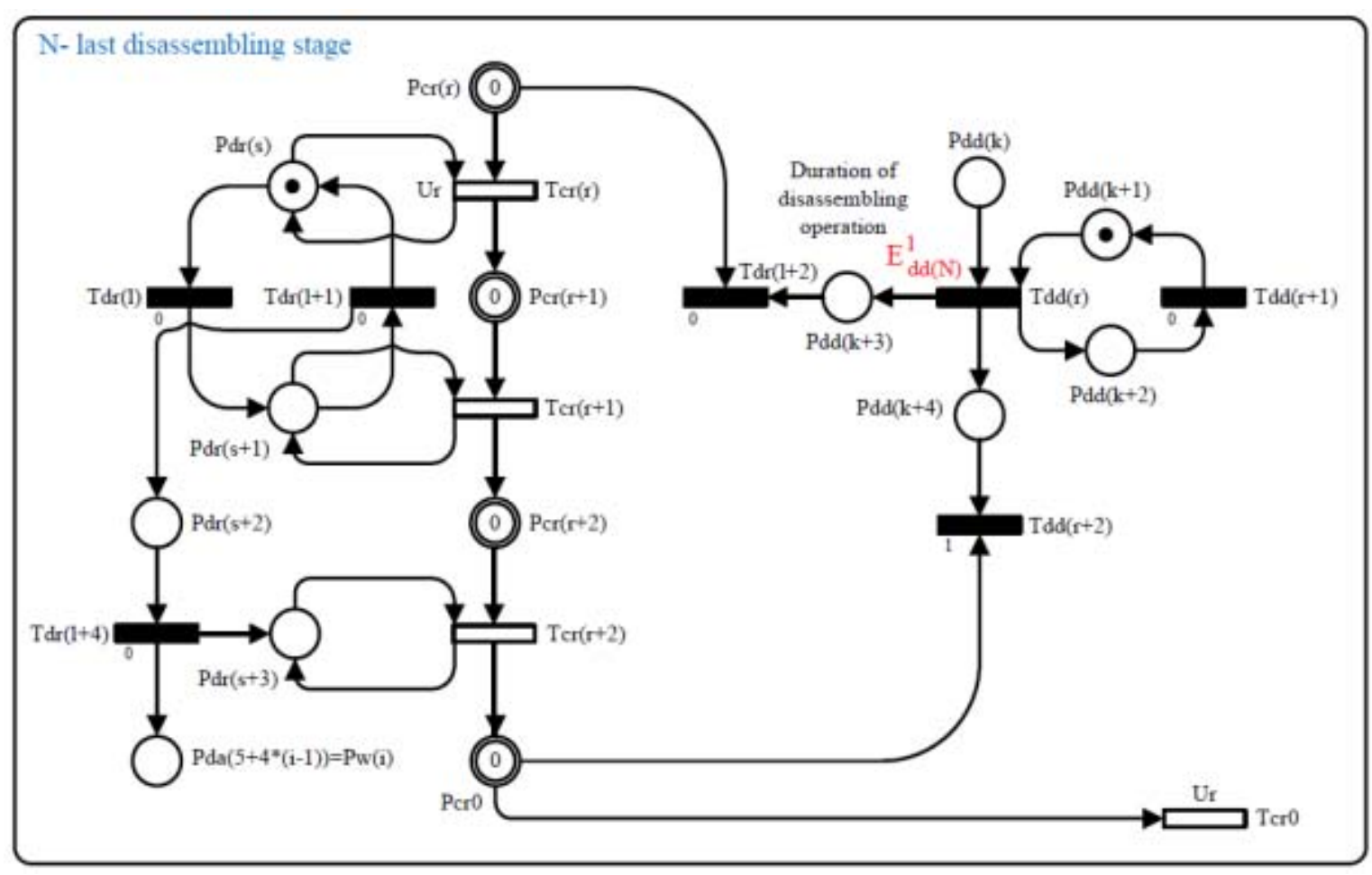

Figure 4. SHPN model of the last disassembly operation, $j=N$ 


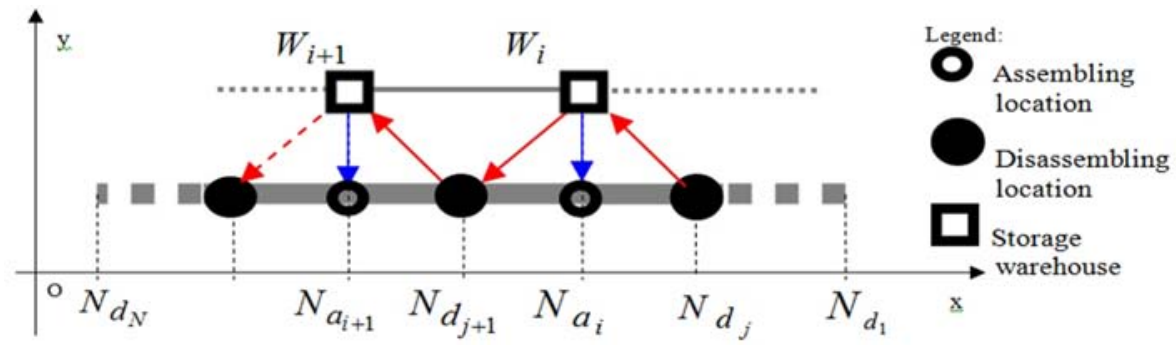

Figure 5. Assembly/Disassembly and storage warehouse locations.

It considers the following notations (Figure 5):

- $\quad N_{a_{i}}, i=\overline{1, N}$ - assembly locations on the positive sense of $O x$ axis.

- $\quad N_{d_{j}}, j=\overline{1, N}$ - disassembly locations on the inverse sense of $O x$ axis. Obviously, $i=N-j+1$.

- $\quad W_{i} ; i=\overline{1, N}$ - warehouse locations, which are identically with the assembly locations. Obviously, $W_{N+1-j} \equiv W_{i}, j=\overline{1, N}$

- $\quad D\left(N_{d_{j}}, W_{N+1-j}\right)$ - distance between disassembly location $N_{d_{j}}$ and the corresponding storage warehouse $W_{N+1-j}$

- $\quad D\left(W_{N+1-j}, N_{d_{j+1}}\right)$ - distance between last storage warehouse $W_{N+1-j}$ and the next disassembly location $N_{d_{j+1}}$.

- $\quad D_{r_{j}}=D\left(N_{d_{j}}, W_{N+1-j}\right)+D\left(W_{N+1-j}, N_{d_{j+1}}\right) \quad$ distance travelled by the mobile robot in the $j$ stage of disassembly.

- $\quad r=1+(j-1) \cdot 3$ - indexes a continuous place of the robot states, $\mathrm{Pcr}$; a continuous transition of the robot, Tcr and a discrete transition of disassembly process $T d d$.

- $\quad k=1+(j-1) \cdot 5$ - indexes a discrete place of disassembly process, $P d d$.

- $\quad l=1+(j-1) \cdot 4$ - indexes a discrete place of the robot states, $T d r$.

\section{Generalized Model based Control of A/DML Served by WMR}

\subsection{The formalism of SHPN model}

The SHPN model associated to A/DML is a triplet
$S H P N=\langle T H P N, E$, Sync $\rangle$

such that: THPN is a seventhly

$T H P N=\left\langle P, T\right.$, Pre, Post $, m_{0}, h$, tempo $\rangle$

$E$ is a set of external events

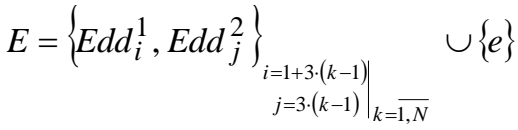

Sync is a function from the set of the discrete disassembly transitions to the set of external events

$$
\text { Sync: } T \rightarrow\left\{E^{1}, E^{2}\right\} \cup\{e\}
$$

where $e$ is the always occurring event (it is the neutral element of the monoid $E^{*}$ ) and

$$
\begin{aligned}
& \text { Sync: }\left\{\operatorname{Tdd}_{r}\right\}_{r=1+3 \cdot(k-1)_{k=1, N}} \rightarrow\left\{E^{1}, E^{2}\right\} \\
& \text { Sync: }\left\{\operatorname{Tdd}_{i}\right\}_{i=3 \cdot(k-1)_{k=2, N}} \rightarrow\left\{\operatorname{Edd}_{i}^{2}\right\}_{i=3 \cdot(k-1)_{k=2, N}} \\
& \text { Sync: } T \backslash\left\{\operatorname{Tdd}_{r}\right\}_{r=1,3+3 \cdot(N-1)} \cup\left\{T d r_{l}\right\}_{l=\overline{1,4+5 \cdot(N-1)}} \\
& \cup\left\{\operatorname{TCr}_{r}\right\}_{r=1,3+3 \cdot(\mathrm{N}-1)} \rightarrow e \\
& P=\left\{P_{1}, P_{2}, \ldots P_{n}\right\}=P^{D} \cup P^{C}
\end{aligned}
$$

is a finite, not empty, set of places with $P^{D}$ the set of discrete places

$$
\begin{aligned}
P^{D} & =\{P d q\}_{i=1,13+4 \cdot(N-1)} \cup\left\{P d d_{r}\right\}_{r=1,5+5 \cdot(N-1)} \\
& \cup\left\{P d r_{s}\right\}_{s=1,4+8(N-1)}
\end{aligned}
$$

and $P^{C}$ the set of continuous places

$$
P^{C}=\left\{\mathrm{Pcr}_{k}\right\}_{k=0,3+3(N-1)},
$$

where:

- $\left\{P d a_{i}\right\}$ is the set of discrete places for assembly process;

- $\left\{P d d_{j}\right\}$ is the set of discrete places for disassembly process;

- $\left\{P d r_{k}\right\}$ is the set of discrete places for the 
states of mobile robot while serving disassembly process;

- $\left\{\mathrm{PCr}_{k}\right\}$ is the set of continuous places associated to the distances performing by the mobile robot for each disassembly operation in order to transport the disassembled component from the disassembled location to the storage location;

$T=\left\{T_{1}, T_{2}, \ldots, T_{m}\right\}=T^{D} \cup T^{C}$

is a finite, not empty, set of transitions with $T^{D}$ the set of discrete transitions

$$
\begin{aligned}
T^{D}= & \left\{\operatorname{Tda}_{i}\right\}_{i=1, \overline{1,7+2 \cdot N}} \cup\left\{\operatorname{Tdd}_{r}\right\} r=\overline{1,3+3 \cdot(N-1)} \\
& \cup\left\{T d r_{l}\right\}_{l=\overline{1,4+5 \cdot(N-1)}}
\end{aligned}
$$

and $T^{C}$ the set of continuous transitions

$$
T^{C}=\left\{\operatorname{Tcr}_{r}\right\}_{r=1,3+3 \cdot(N-1)}
$$

where:

- $\quad\left\{T d a_{i}\right\}$ is the set of discrete transitions for assembly operations model

- $\quad\left\{T d d_{j}\right\}$ is the set of discrete transitions for disassembly operations model;

- $\quad\left\{T d r_{k}\right\}$ is the set of discrete transitions for states of mobile robot while serving disassembly operations;

- $\left\{T c r_{k}\right\}$ is the set of continuous transitions associated to distances performing by the mobile robot for each disassembly operation. To these transitions is associated the maximum linear speed of the WMR.

Pre: $P \times T \rightarrow Q_{+}$or $N$ is the input incidence application;

Post : $P \times T \rightarrow Q_{+}$or $N$ is the output incidence application; $m_{0}: P \rightarrow R_{+}$or $N$ is the initial marking;

$h: P \cup T \rightarrow\{D, C\}$

called "hybrid function", indicates for every node whether it is a discrete node (sets $P^{D}$ and $T^{D}$ ) or a continuous one (sets $P^{C}$ and $T^{C}$ ),

$$
\begin{aligned}
& h: P^{D} \cup T^{D} \rightarrow\{D\} \\
& h: P^{C} \cup T^{C} \rightarrow\{C\}
\end{aligned}
$$

tempo is a function from the set $T$ of transitions to the set of positive or zero rational numbers,

tempo: $T \rightarrow Q_{+} \cup\{0\}$

If $T_{j} \in T^{D}$, then $d_{j}=\operatorname{tempo}\left(T_{j}\right)$ is timing associated with $T_{j}$. For each discrete assembly transition of the set

$$
\begin{aligned}
& T_{a}^{D}=\left\{T d a_{i}\right\}_{i=2 \cdot k_{k=1, N}} \cup\left\{T d a_{2 \cdot(N+1)}\right\} \\
& \operatorname{tempo}\left(T d a_{i}\right)=d_{d a_{i}}
\end{aligned}
$$

where $d_{d a_{i}}$ represents the duration (in seconds) associated to the corresponding assembly operation. For each discrete disassembly transition of the set

$T_{d}^{D}=\left\{T d d_{r}\right\}_{r=1+3 \cdot(k-1)_{k=1, N}}$,

$d_{d d_{r}}$ is the duration of the corresponding disassembly operation. For each discrete WMR transition of the set

$T_{r}^{D}=\left\{T d r_{l}\right\}_{l=4+5 \cdot(k-2)_{k=2, N}}$,

$d_{d r_{l}}$ is the duration of RM positioning in picking up and dropping down for a disassembled component.

If $T_{c r} \in T^{C}$ then

$$
U_{r}=\frac{1}{\operatorname{tempo}\left(T_{c r}\right)}
$$

is flow rate associated to $T_{c r}$.

For

$$
T^{C}=\left\{\operatorname{Tcr}_{r}\right\}_{r=3+3 \cdot(k-1)_{k=1, N}}
$$

$U_{c r_{r}}=U_{r} ; U_{r \max }=V_{r}$ where $U_{c r}$ is the variable flow of mobile robot displacement between disassembly stations. Consider the average speed of motion of WMR, $V_{r}=94 \mathrm{~mm} / \mathrm{s}$.

Definition 1: The ED-enabling degree of a Ctransition $T_{j}$ for a marking $m$, denoted by $E D\left(T_{j}, m\right)$, is the enabling degree of $T_{j}$ after all the arcs, from a C-place to a C-transition, have been removed:

$$
E D\left(T_{j}, m\right)=\min _{P_{i} \in{ }^{0} T}\left(\frac{m_{i}}{\operatorname{Pre}\left(P_{i}, T_{j}\right)}\right)
$$


Definition 2: The maximal firing speed of transition $T_{c r_{r}}$ is the product of its flow rate $U_{r}$ by its $E D$ - enabling degree. Suitable definitions 1 and 2, for the general case, it can write:

$$
\begin{aligned}
& E D\left(T_{c r_{j}}, m_{c r}(j+1)\right)=\{0,1\} \\
& m_{c r}(j+1)=V_{j} \cdot w\left(T c r_{j} \times P c r_{j+1}\right) \\
& w\left(T c r_{r} \times P c r_{r+1}\right)= \\
& =D\left(W_{N+1-j}, N_{d_{j+1}}\right) / D\left(N_{d_{j}}, W_{N+1-j}\right)
\end{aligned}
$$

where $m_{c r}(j+1)$ is the mark associated to a continuous place and $w\left(\operatorname{Tcr}_{(r)} \times \operatorname{Pcr}_{(r+1)}\right)$ is the weight of the arc from a continuous transition to a continuous place of the WMR [9]. The analysis of SHPN model is relevant at the basic level accordingly with an elementary THPN module, denoted e-THPN. SHPN model is obtained by recurrent assembling of these elementary eTHPN modules (corresponding to each basic disassembly served by WMR with RM).

\subsection{SHPN model customized for HERA \& Hortsmann mechatronic line}

General approach will customize (Figure 9) to an A/DML didactic mechatronics line, HERA
\& Horstmann, shown in Figure $6 a$ and 6b, which makes assembling a piece of five components, shown in Figure 6c and Figure 6d. Flexible line includes five individual workstations with different tasks, carrying and transporting, pneumatic workstations, conveyor belt, sorting unit, test station and warehouse. The assembly/disassembly manufacturing flexible line is equipped with SIEMENS Simatic S7-300 PLC (Programmable Logic Controller), with 5 distributed modules connected by Profibus.

The WMR, Pioneer 3-DX, is with two driving wheels and one rear wheel, has its own odometric system and an on-board embedded microcontroller is able to read the position information and to send it, over a WI-FI link, to a remote PC where runs the according to a specific protocol and send the data to PLC of the assembly line.

In Figure 7 is presented the schematic representation of the HERA \& Hortsmann didactic platform destinated to a particular assembly/disassembly product comprised of 5 parts, served by WMR with RM. WMR carries the component from the place where disassembly occurs to the appropriate storage.
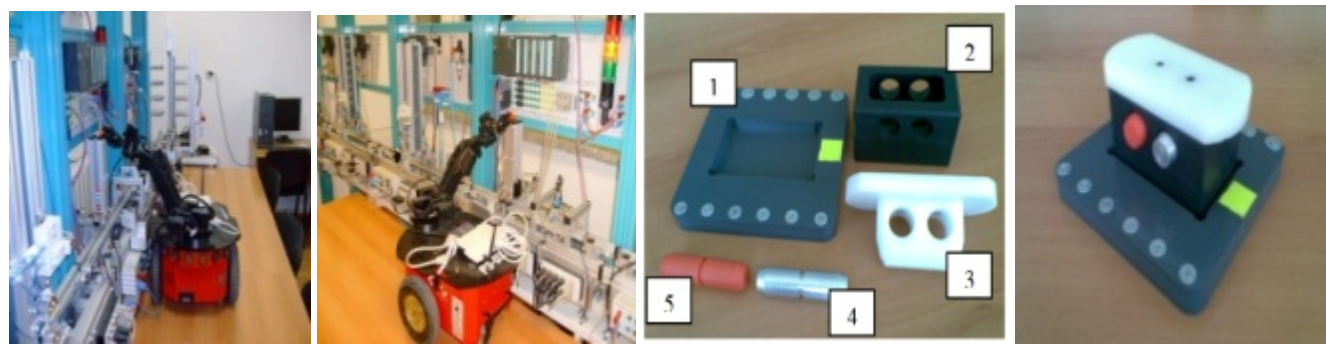

Figure 6. a) and b) assembly line, Hera, served by WMR, Pioneer 3-DX, equipped with RM, Pioneer 5-DOF Arm; c) parts; d) assembled product

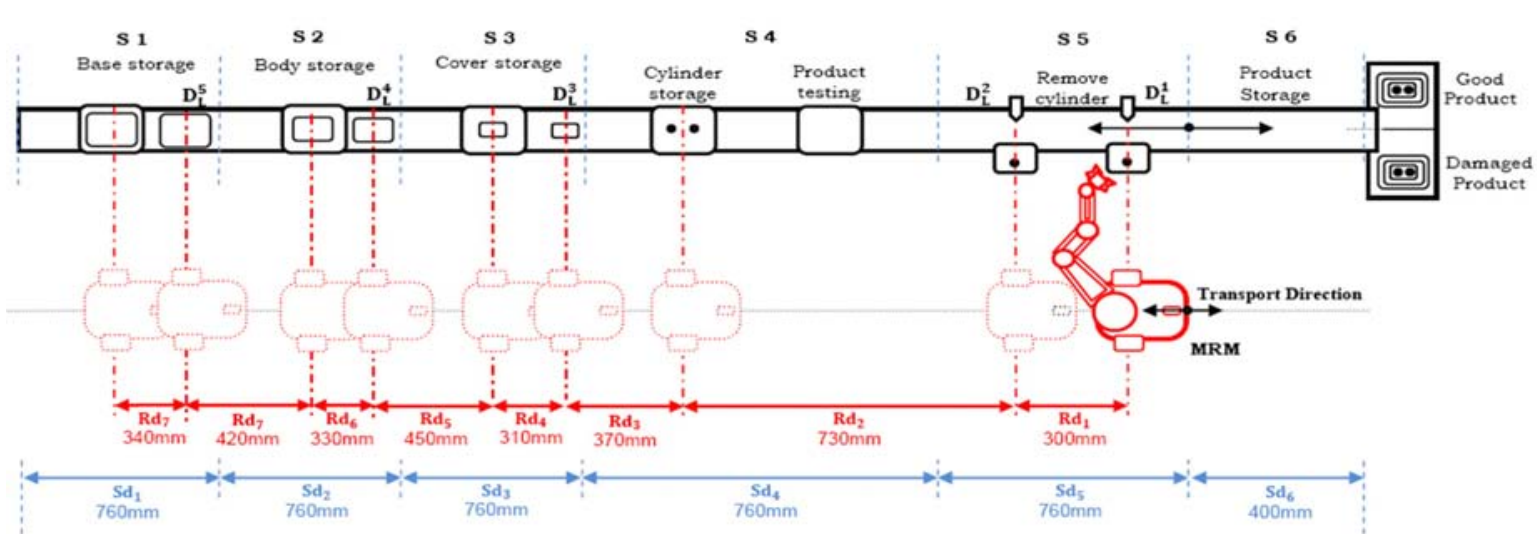

Figure 7. Assembly/disassembly line of a product consists of 5 components, served by the WMR equipped with RM 


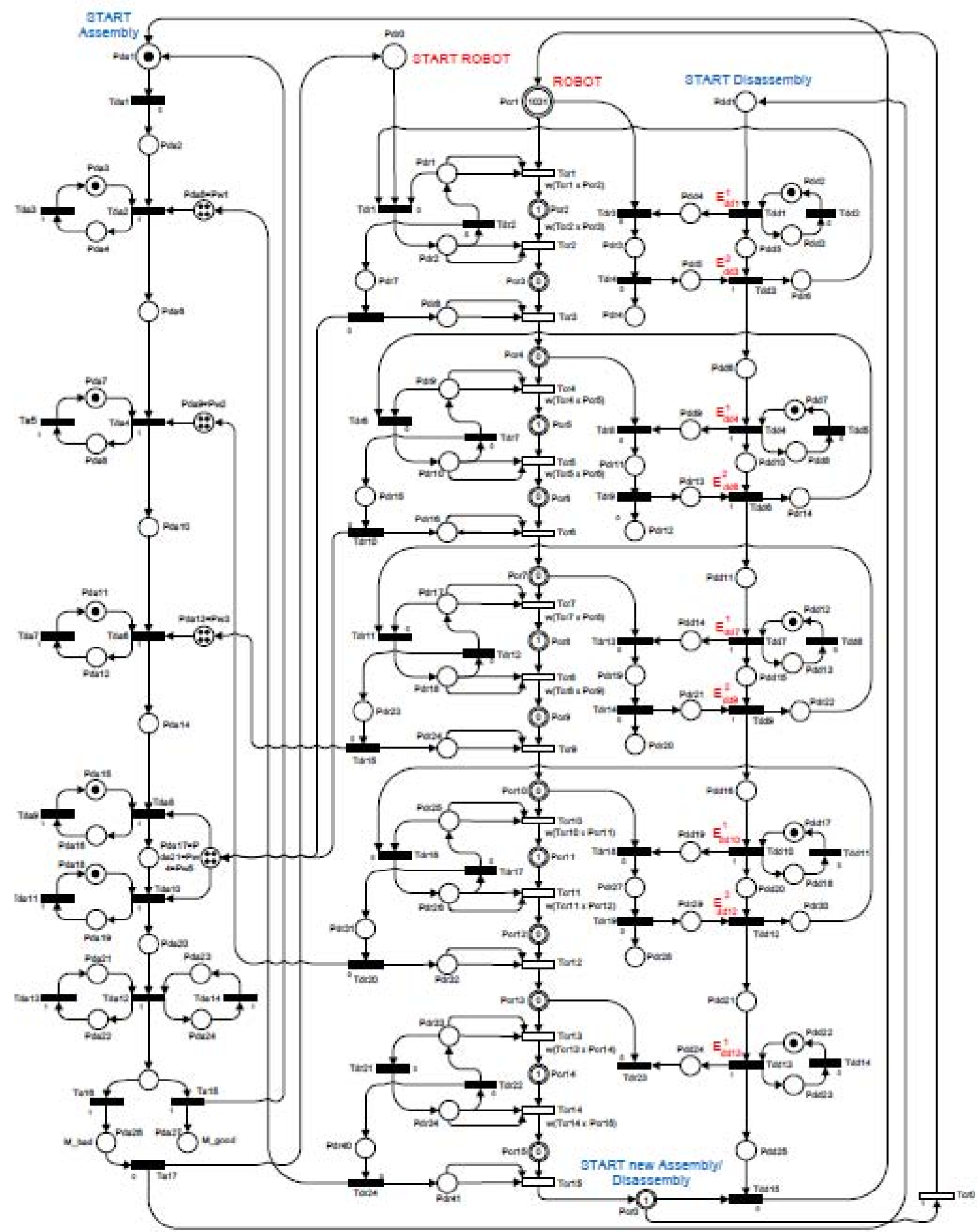

Figure 9. SHPN model cooresponding of reversible assembly/disassembly manufacturing line (A/DML) served by wheeled mobile robot (WMR) equipped with robotic manipulator (RM), composed by 5 workstations

For $N=5$ (A/DML HERA \& Horstmann), the SHPN model become:

$$
\begin{array}{ll}
\text { For } N=5(\text { A/DML HERA \& Horstmann), the } & T^{C}=\left\{T c r_{k}\right\}_{k=1,15} \\
\text { SHPN model become: } & \\
P^{D}=\left\{P d a_{i}\right\}_{i=1,29} \cup\left\{P d d_{j}\right\}_{j=1,25} \cup\left\{P d r_{k}\right\}_{k=1,41} & T_{a}^{D}=\left\{T d_{i}\right\}_{i=\{2,4,6,8,10\}} \cup\left\{T d a_{12}\right\} \\
P^{C}=\left\{P c r_{k}\right\}_{k=\overline{0,15}}, & \operatorname{tempo}\left(\operatorname{Tda}_{i}\right)_{i=\{2,4,6,8,10,12\}}=\{9.5,9.3,8.5,0.5,4.75,27.2\}
\end{array}
$$$$
P^{C}=\left\{P c r_{k}\right\}_{k=0,15} \text {, }
$$$$
T^{D}=\left\{T d a_{i}\right\}_{i=1,17} \cup\left\{\operatorname{Tdd}_{j}\right\}_{j=1,15} \cup\left\{\operatorname{Tdr}_{k}\right\}_{k=\overline{1,24}}
$$ 
where $d_{d a_{i}}$ represents the duration of the current assembly operation together with the transport time to the next assembly location, for $i=\{2,4,6,8,10\}$ and the duration of the quality test together with the transport time to the elevator of end products warehouse, for $i=\{12\}$;

$T_{d}^{D}=\left\{T d d_{r}\right\}_{r=\{1,4,7,10,13\}}$

tempo $\left(T d d_{r}\right)_{r=\{1,4,7,10,13\}}=\left(d_{d d_{r}}\right)_{r=\{1,4,7,10,13\}}=1$

$T_{r}^{D}=\left\{T d r_{l}\right\}_{l=\{4,9,14,19\}}$

tempo $\left(T d r_{l}\right)_{l=\{4,9,14,19\}}=\left(d_{d r_{l}}\right)_{l=\{4,9,14,19\}}$

$\left(d_{d r_{l}}\right)_{l=\{4,9,14,19\}}=\{5.1,21.2,8.9,7.8\}$

Sync : $\left\{\text { Tdd }_{j}\right\}_{j=\{1,3,4,6,7,9,12,13\}} \rightarrow\left\{\right.$ Edd $\left.^{1}, E_{d d^{2}}\right\}$

where:

Sync : $\left\{\operatorname{Tdd}_{i}\right\}_{i=\{1,4,7,13\}} \rightarrow\left\{\operatorname{Edd}_{i}^{1}\right\}_{i=\{1,4,7,13\}}$;

Sync : $\left\{\operatorname{Tdd}_{i}\right\}_{i=\{3,6,9,12\}} \rightarrow\left\{\operatorname{Edd}_{i}^{2}\right\}_{i=\{3,6,9,12\}}$;

Sync : $\left\{\operatorname{Tdd}_{j}\right\}_{j=1, \overline{16}} \cup\left\{T d r_{k}\right\}_{k=1,21} \cup\left\{T c r_{k}\right\}_{k=1, \overline{10}} \rightarrow e$

Consider the average speed of motion of WMR, $V_{r}=94 \mathrm{~mm} / \mathrm{s}$. For $N=5$ (Figure 9) the arch $\left(P_{i} \times T_{j}\right)$, where $P_{i} \in\left\{T c r_{k}\right\}_{k=\overline{1,10}} \cap P^{D}$, have the weight equal to one. Therefore,

$V c r_{k}=U c r_{k}=\left.2\right|_{k=1,10}$.

In Figure 8 is shown the simulated response of the continuous and discrete places of WMR with RM of SHPN model from Figure 9.
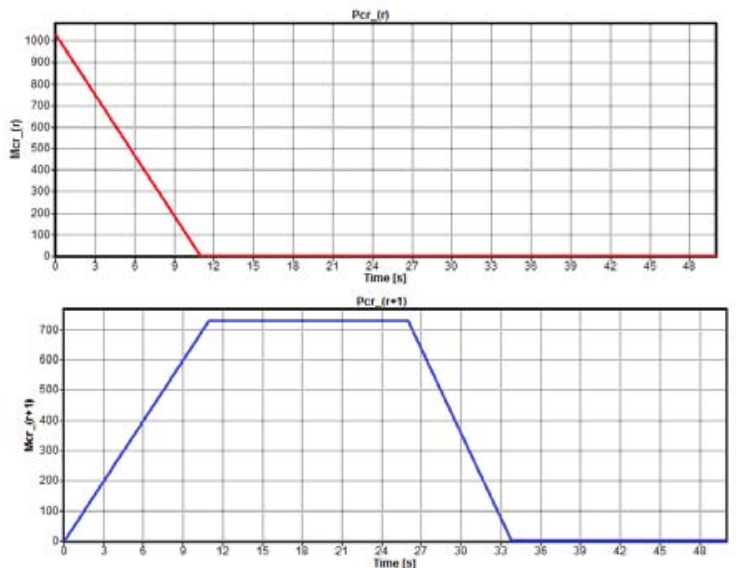
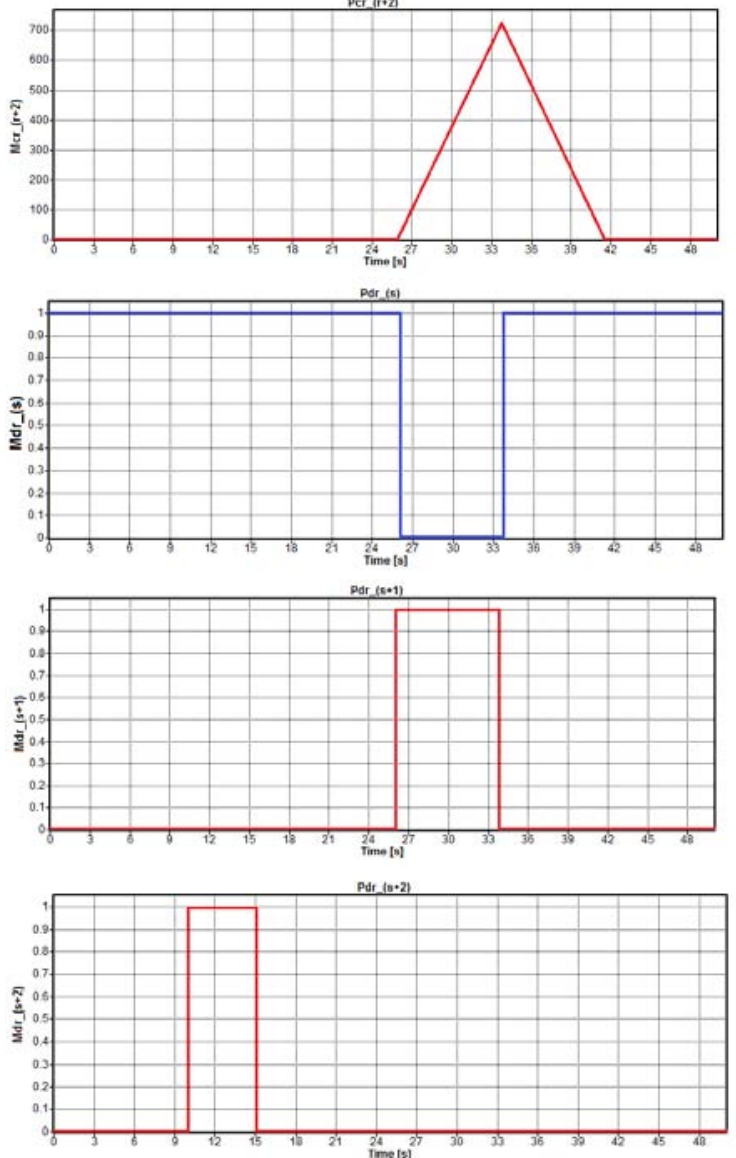

Figure 8. Variation of the continuous and discrete places asociated to displacements of WMR and RM corresponding to the $\mathrm{j}$ - stage of disassembling.

\section{Time Cycle Optimization for Disassembling Operations}

Within the e_SHPN network, the weight of the arcs $\quad w_{r}=\left.\left(T c r_{r} \times P c r_{r}\right)\right|_{r=1+3 \cdot(j-1)_{j=1, N}} \quad$ and

$w_{r+1}=\left.\left(T c r_{r+1} \times P c r_{r+1}\right)\right|_{r=1+3 \cdot(j-1)_{j=1, N}} \quad, \quad$ for $r=1+3 \cdot(j-1)_{j=1, \bar{N}}$ are:

$\left\{\begin{array}{l}w_{r}=D\left(w_{N+1-j}, N_{d_{j+1}}\right) / D\left(N_{d_{j}}, W_{N+1-j}\right) \\ w_{r+1}=D\left(N_{d_{j+1}}, W_{N-j}\right) / D\left(W_{N+1-j}, N_{d_{j+1}}\right)\end{array}\right.$

The elementary time cycle (ETC) for the mobile robot (corresponding to the e_SHPN model, stage "j") is the travel duration with constant speed between storage warehousedisassembling location added to durations of disassembly and manipulation operations performed by mobile robot:

- the duration of the disassembly operation corresponding to "j" stage of disassembly $\left(d_{d d_{r}}\right)$; 
- the travel duration: workstation for disassembling - storage warehouse $D\left(N_{d_{j}}, W_{N+1-j}\right) / V r$; storage warehouse next disassembly workstation $D\left(W_{N+1-j}, N_{d_{j+1}}\right) / V r$.

- the picking-up and dropping-down durations for a disassembled component, followed by gripper closing $\left(d_{d r_{1}}\right)$

The time cycle duration and its values components can be identified within the temporal marking evolution (Figure 8) corresponding to " $\mathrm{j}$ " stage of disassembly in SHPN model. In this case the ETC for the mobile robot for " $\mathrm{j}$ " stage, are:

$$
\begin{aligned}
T_{E T C} & =D\left(N_{d_{j}}, W_{N+1-j}\right) / V_{r}+d_{d d_{j}} \\
& +d_{d r_{j+3}}+D\left(W_{N+1-j}, N_{d_{j+1}}\right) /\left.V_{r}\right|_{j=1, N}
\end{aligned}
$$

Identifying these variables in SHPN model, this relationship becomes:

$$
\begin{aligned}
T_{E T C} & =\left(m_{c r_{r+1}}\right) / V r=\left(m_{c r_{r}}\right) / V r+d_{d d_{j}} \\
& +d_{d r_{j+3}}+\left(m_{c r_{r+2}}\right) /\left.V_{r}\right|_{r=1+3 \cdot(j-1)_{j=1, N}}
\end{aligned}
$$

where, for $r=1+3 \cdot(j-1)_{j=1, N}$ :

$$
\begin{aligned}
& m_{c r_{r}}=D\left(N_{d_{j}}, W_{N+1-j}\right) \\
& m_{c r_{r+2}}=m_{c r_{r}} \cdot w_{r} \cdot w_{r+1} \\
& m_{c r_{r+2}}=D\left(W_{N+1-j}, N_{d_{j+1}}\right)
\end{aligned}
$$

The optimization of time cycle (TCO) for mobile robot implies the minimization of disassembly operations duration (if possible) and the minimization of manipulation durations:

$$
\begin{aligned}
\text { TCO } & =\sum_{j=1}^{N} D\left(N_{d_{j}}, W_{N+1-j}\right) / V_{r} \\
& +\left.\min \left(\sum_{r} d_{d d_{r}}+\sum_{l} d_{d r_{l}}\right)\right|_{\substack{r=1+3 \cdot(k-1)_{k=\overline{1, N}} \\
l=4+5 \cdot(k-2)_{k=2, N}}} \\
& +\sum_{j=1}^{N} D\left(W_{N+1-j}, N_{d_{j+1}}\right) / V_{r}
\end{aligned}
$$

At the same time it must be provided the temporal synchronization between ETC and the disassembly duration added to time travel product between two successive workstations. Within the SHPN model this restriction is equivalent to the avoidance condition for PN model blockage:

$$
\left\{\begin{aligned}
T C O= & \sum_{j=1}^{N} D\left(N_{d_{j}}, W_{N+1-j}\right) / V_{r} \\
& +\left.\min \left(\sum_{r} d_{d d_{r}}+\sum_{l} d_{d r_{l}}\right)\right|_{\substack{r=1+3 \cdot(k-1)_{k=1, N} \\
l=4+5 \cdot(k-2)_{k=2, N}}} \\
& +\sum_{j=1}^{N} D\left(W_{N+1-j}, N_{d_{j+1}}\right) / V_{r} \\
\min \left(d_{d d_{r}}\right. & \left.+d_{d r_{l}}\right)+D\left(W_{N+1-j}, N_{d_{j+1}}\right) /\left.V_{r}\right|_{\substack{r=1+3 \cdot(k-1)_{k=1, N} \\
l=4+5 \cdot(k-2)_{k=2, N}}} \\
& \leq d_{d d_{r}}+\left.d_{d d_{r+1}}\right|_{r=1+3 \cdot(k-1)_{k=1, N}}
\end{aligned}\right.
$$

\section{Conclusions}

A SHPN model, in synchronized form, based real-time control of fully reversible assembly/disassembly mechatronics line is presented in this paper. The SHPN model has been conditioned on certain state transitions by external events representing signals supplied by sensors. The A/DML is served by a WMR equipped with RM which is used only in disassembling in order to transport the disassembled components to the storage warehouses. Therefore, the assembly line becomes reversible, i.e. executes automated disassembly. A disassembly process is started when the final product, obtained by assembly, fails quality test. The hybrid control system takes into account the distribution of the necessary tasks to perform the disassembly of components by using the robot synchronization with the A/DML. An optimization approach of time cycle for repetitive processes is proposed.

\section{Acknowledgements}

This work was supported by UEFISCDI, project number PN-II-ID-PCE-2011-3-0641

\section{REFERENCES}

1. BALDWIN, D. F., T. E. ABELL, C. M. LUI, T. L. DE FAZIO, D. E. WHITNEY, An Integrated Computer Aid for Generation and Evaluation Assembly Sequences for Mechanical Products, IEEE Transactions on Robotics until Automation, 1991, pp. 78-94.

2. CHOI, C. K., X. F. ZHANG, T. L. NG, W. S. LAU, On the Generation of Product 
Assembly Sequences, International Journal of Production Research, 1998, pp. 617-633.

3. DAVID, R., H. ALLA, Discrete, Continuous and Hybrid Petri Nets, ISBN 978-3-642-10668-2, Springer Verlag, Berlin Heidelberg, 2010.

4. GANGET, J., G. HATTENBERGER, R. ALAMI, Task Planning and Control for Multi-UAV System: Architecture and Algorithms, IEEE International Conference on Intelligent Robot and System, vol. 18, 2005, pp. 758-768.

5. SELIGER, G., W. GRUDZIEN, H. ZAIDI, New Methods of Product Data Provision for a Simplified Disassembly, Procs. of the 6th International Seminar on Life Cycle Engineering, Kingston, Canada, June 21-23, 1999. pp. 250-259.

6. MINCA, E., A Theoretical Approach of the Time Cycle Optimisation Based Control of a Mechatronics Line Served by Mobile Robot, Applied Mechanics and Materials, Trans Tech Publications, Switzerland, vols. 321-324 (2013) pp 16661669.

7. RADASCHIN, A., A. FILIPESCU, V. MINZU, E. MINCA, A. JR. FILIPESCU, Adaptive Disassembly Sequence Control by Using Mobile Robots and System Information, Proceeding of 15th IEEE International Conference in System Theory, Control and Computing, 14-16 Oct., 2011, Sinaia, Romania, pp. 499-505, ISBN: 978973-621-323-6.
8. RADASCHIN, A., A. VODA, E. MINCA, A. FILIPESCU, Task Planning Algorithm in Hybrid Assembly/Disassembly Process, 14th IFAC Symposium on Information Control Problems in Manufacturing, May 23-25, 2012, Bucharest, pp. 571-576, ISSN: 1474-6670; ISBN: 978-3-902661-98-2.

9. IACOB, R., D. POPESCU, Generation of Disassembly Directions Based on Component Mobility, Studies in Informatics and Control, ISSN 1220-1766, vol. 22(4), 2013, pp. 307-318.

10. KOPACEK, B., P. KOPACEK, Robots for Disassembly, Proceedings of the 30th International Symposium on Robotics, Tokyo, pp. 207-212.

11. DAVID, R., H. ALLA, Discrete, Continuous and Hybrid Petri Nets, ISBN 978-3-642-10668-2, Springer-Verlag Berlin Heidelberg, 2010.

12. FILIPESCU, A., S. FILIPESCU, E. MINCA, Hybrid System Control of an Assembly/Disassembly Mechatronic Line Using Robotic Manipulator Mounted on Mobile Platform, The 7th IEEE Conference on Industrial Electronics and Applications (ICIEA2012), 18-20 July, 2012, Singapore, pp. 433-438, IEEE Catalog Number CFP 1220A-CDR, ISBN: 978-1-4577-2117-5. 